Ann. Biol. anim. Bioch. Biophys., I976, 16 (4), 56r-568.

\title{
ÉTUDE HISTOLOGIQUE DU PLACENTA DE BREBIS ET DE CHEVRE AU MOMENT DE LA PARTURITION
}

\author{
Nicole MAURIN et M.-J. BOSC* \\ Laboratoire d'Histologie, \\ Faculté de Médecine Saint-Antoine, \\ 27, rue Chaligny, \\ 75571 Paris Cedex 12 \\ * Station de Physiologie de la Reproduction, \\ Centre de Recherches de Tours, I. N.R.A., \\ Nouzilly, 37380 Monnaie
}

\section{RÉSUMÉ}

Chez la Brebis et chez la Chèvre, l'induction de la parturition par la Dexaméthasone ne semble pas modifier l'évolution ultrastructurale du placenta et de l'endomètre. Cette évolution des tissus placentaires apparaît au cours du dernier jour de la gestation.

Il se produit, du côté maternel, une dissociation entre le conjonctif dense du "berceau " du placentôme et le conjonctif lâche utérin, associée à une altération de la vascularisation villositaire ; du côté fœetal on observe simultanément une multiplication des structures de type lysosomal et la dégénérescence de quelques cellules. Ces changements s'accentuent très rapidement après la naissance, avant l'expulsion du placenta. On constate la présence de polynucléaires dans les vaisseaux sanguins du tissu conjonctif utérin qui est devenu légèrement cedémateux. L'expulsion du placenta laisse des bribes de tissu fœtal dans les touffes caronculaires ; leur désengrènement est facilité par le développement de l'œdème qui provoque l'éversion du caroncule.

Depuis que le rôle des corticostéroïdes foetaux a été mis en évidence dans le déclenchement de la parturition chez la Brebis et la Chèvre (LigGins et al., I973; ThORBURN et al., 1972), des méthodes ont été mises au point pour induire la mise bas par des composés de synthèse (Bosc, I974). Si, chez ces espèces, l'induction du part n'est suivie d'aucun effet secondaire, chez la Vache par contre, elle s'accompagne d'une augmentation importante du taux des rétentions placentaires (Bosc, 1974). Ceci suggère que les facteurs dont dépend la mise bas ne correspondent pas nécessairement à ceux qui déterminent l'expulsion du placenta.

Peu de descriptions du placenta des ruminants domestiques ont été réalisées à la fin de la gestation (Drieux et ThIERry, I95I $a, b$; Pavsic et Skuser, r97I ; 
STEVEN, I975). Aussi avons nous entrepris de rechercher les modifications cytologiques éventuelles qui apparaissent dans les tissus maternels et foetaux au niveau du placentôme et de la zone intercotylédonnaire de la Brebis et de la Chèvre dans les heures qui précèdent ou qui suivent la délivrance du placenta. A titre de comparaison et pour essayer de préciser la chronologie des prélèvements par rapport au terme, quelques mises bas ont été induites par la déxaméthasone.

\section{CONDITIONS EXPÉRIMENTALES}

Nous avons utilisé des Brebis Ile-de-France et des Chèvres Alpines et Poitevines dont Ia date d'insémination était connue. Les Brebis de cette race ont une durée moyenne de gestation de 148 jours et les premiers agnelages se produisent le $144^{\mathrm{e}}$. Les Chèvres ont une durée moyenne de gestation de 150 jours, fortement influencée par la prolificité (ASDELL, I964).

Ces animaux ont été répartis en 3 groupes, suivant qu'ils ont été abattus plus de 28 heures avant le part (Groupe 1 ), dans les 28 heures précédant la mise bas (Groupe 2) ou après celle-ci (Groupe 3), selon le tableau ci-dessous :

Chronologie des prélèvements placentaives

\begin{tabular}{|c|c|c|c|c|c|c|c|c|c|c|c|}
\hline $\begin{array}{l}\text { Heures } \\
\text { Part }=-\infty\end{array}$ & -96 & -66 & -28 & -12 & 0 & $+1 \mathrm{~h} 30$ & +24 & +36 & +42 & +72 & $+9 \geq$ \\
\hline Chèvres $\left\{\begin{array}{l}\text { Témoins } \\
\text { Traitées }\end{array}\right.$ & 1 & & -2 & 1 & & 1 & 1 & & 1 & 1 & \\
\hline Brebis $\left\{\begin{array}{l}\text { Témoins } \\
\text { Traitées }\end{array}\right.$ & & 3 & 1 & 1 & & 1 & 1 & 1 & & 1 & 1 \\
\hline
\end{tabular}

Des animaux des groupes 2 et 3 ont été traités avec I6 mg de Dexaméthasone par voie intramusculaire afin d'induire le part qui survient $4^{\circ}$ heures en moyenne après le traitement (Bosc I974).

A l'abattage, des prélèvements de placentôme et de zone intercotylédonnaire ont été faits dans les 2 minutes suivant l'extirpation de l'utérus et fixés au mélange de Bouin-Hollande acétique ou de Stieve. Après deshydratation et inclusion à la paraffine, ils sont coupés à $5 \mu \mathrm{m}$ d'épaisseur et colorés au Cleveland-Rücker-Wolfe ou à 1'acide periodique Schiff-hématoxyline de Groat.

Au même moment des fragments des mêmes zones ont été fixés dans du glutaraldéhyde à 4 p. roo dans du tampon phosphate $0,2 \mathrm{M}$ pendant I heure. Après rinçage au tampon phosphate $0,2 \mathrm{M}$, ils ont été post-fixés pendant $\mathrm{I}$ heure dans de l'acide osmique à 2 p. Ioo dans le même tampon puis deshydratés à l'éthanol, rincés à l'oxyde de propylène et inclus dans de l'épon. Les coupes de 600 à $900 \mathrm{~nm}$ d'épaisseur ont été colorées à l'acétate d'uranyle-citrate de plomb.

\section{RÉSULTATS}

\section{I. - Plus de 28 heures avant la parturition (Groupe $\mathrm{r}$ )}

a) La zone intercotylédonnaire, au niveau de laquelle les tissus maternels et foetaux sont simplement apposés, sépare les placentômes les uns des autres. A ce niveau, le tissu maternel est constitué d'un épithélium et d'un tissu conjonctif sous-jacent 
reposant sur le myomètre. Les cellules épithéliales sont organisées en une assise simple prismatique. Leur apex est garni de microvillosités en " bordure en brosse " à la base desquelles se trouvent des vésicules de pynocytose. Les membranes cytoplasmiques sont très interdigitées. Dans le tissu conjonctif, on peut distinguer d'une part une assise sous-épithéliale peu épaisse et riche en fibrocytes, en fibres de réticuline et de collagène et, d'autre part, au contact du muscle utérin, une assise large, riche en substance fondamentale. Cette dernière contient des glandes utérines actives qui débouchent dans des cryptes formées par les replis de l'épithélium utérin.

Le tissu fotal apposé contre l'épithélium utérin est un allantochorion ou un amniochorion ; il est composé d'un tissu conjonctif lâche, limité du côté des cavités allantoïdienne et amniotique par un épithélium simple cubique ou pavimenteux et, du côté qui fait face au tissu maternel, par un épithélium pluristratifié formé de cellules prismatiques et de cellules géantes. Les cellules prismatiques mononuclées sont au contact du " lait utérin " sécrété par les glandes utérines. Elles portent à leur apex des microvillosités formant une "bordure en brosse "; leur cytoplasme peu dense aux électrons contient de nombreuses mitochondries. Les cellules géantes ovoïdes situées entre les cellules prismatiques sont réparties par plages de forte densité (de 40 à 50 cellules/150 $\mathrm{mm}$ d'épithélium contre $\mathrm{I} / \mathrm{I} 50 \mathrm{~mm}$ dans une autre zone) ; la plupart d'entre elles ( $80 \mathrm{p}$. I0o) sont binucléées.

b) Le placentôme représente la zone d'engrènement intime des tissus maternels et fœetaux. D'un nombre variable d'un animal à l'autre, ils sont d'aspects comparables chez la Brebis et la Chèvre.

Du côté maternel, à sa base, le conjonctif lâche s'amincit et ne contient plus de glandes utérines; il est compris entre l'assise musculaire lisse sous-jacente et un ensemble de fibres conjonctives qui forme le "berceau du cotylédon maternel " et qui représente, à ce niveau une modification du conjonctif dense de la zone intercotylédonnaire. Cet ensemble forme une cupule concave dont les fibres orientées longitudinalement sont associées à des fibrocytes. Sur cette cupule se greffent des villosités à la base desquelles 1'orientation des fibres conjonctives se modifie. Dans les villosités de diamètre peu important, les fibres restent parallèles entre elles et au grand axe de la villosité ; lorsque le tronc villositaire a une base large d'où partent des ramifications, son diamètre paraît diminuer brusquement d'une branche mère à une branche fille, où il n'est que faiblement supérieur à la section du capillaire sanguin qui la parcourt. Ces axes conjonctifs maternels sont recouverts par une lame basale et par un épithélium syncitial en continuité avec l'épithélium utérin. Il présente à sa partie apicale de nombreuses mitochondries et des vésicules de pynocytose ainsi que des microvillosités qui s'entremêlent avec les microvillosités fœatales.

Du côté foetal, les villosités qui s'imbriquent dans les villosités maternelles sont composées d'un axe conjonctif parcouru par un vaisseau sanguin et recouvert de l'épithélium trophoblastique. Cet épithélium a des caractéristiques différentes suivant sa place dans le placentôme :

- D'une part au niveau des arcs villositaires (au sommet du placentôme) cet épithélium baigne dans du sang maternel extravasé. Cette zone, dite "de résorption sanguine ", se présente macroscopiquement sous l'aspect d'une frange annulaire sombre; les cellules géantes n'y sont pas modifiées mais les cellules prismatiques sont le siège d'une intense phagocytose des globules rouges maternels (fig. I). Ces 
cellules, pauvres en organites, contiennent des mitochondries localisées au pôle cellulaire apical, et des corps denses aux électrons ressemblant à des lysosomes; ces "corps lytiques " contribuent activement à la lyse des globules rouges phagocytés à l'apex de la cellule et inclus dans des phagolysosomes; quand il ne reste plus dans cette vésicule que la membrane de l'érythrocyte, le phagolysosome se fragmente en de nombreux saccules peu denses aux électrons qui semblent déverser leur contenu dans l'espace intercellulaire. Le processus de digestion évolue du pôle apical de la cellule vers la zone infranucléaire. Dans les cellules âgées apparaissent des « figures myéliniques " (EDwards et Simon, I970).

- D'autre part à un niveau plus profond de la villosité fœtale, les cellules qui digéraient les globules rouges disparaissent et sont remplacées par des cellules cubiques qui constituent alors environ $90 \mathrm{p}$. Ioo des cellules de la zone d'engrènement. Leur noyau est nucléolé, l'ergastoplasme est le plus souvent organisé en anneaux accompagnés de ribosomes libres; le système golgien, les mitochondries et les vésicules de pynocytose sont regroupées au pôle apical garni de microvillosités qui s'imbriquent dans celles de l'épithélium utérin. Dans cette zone d'engrènement se trouvent les cellules géantes mononucléées ou plurinucléées (Io p. Ioo des cellules environ) ; elles se distinguent par leur taille (Io à $I_{5} \mu \mathrm{m}$ ) et par celle de leur noyau $(6 \mu \mathrm{m})$; leur membrane cytoplasmique n'a pas de microvillosités et n'est jamais en contact direct avec les tissus maternels. La majorité a une polarité marquée car tous leurs organites sont regroupés à l'un de leurs pôles, le reste du cytoplasme étant rempli de granulations de taille et de densité très variable, entourées ou non par une membrane.

\section{II. - Au cours des 28 heures qui précèdent le part (Groupe 2)}

Que ce soient les Brebis ou les Chèvres, aucune différence apparente n'a été observée entre les animaux témoins et traités par la Dexaméthasone.

Du côté maternel il se produit une dissociation discontinue et de largeur variable entre d'une part le conjonctif dense utérin et le conjonctif lâche des glandes utérines et d'autre part entre ce conjonctif lâche et celui du "berceau ". La déchirure est particulièrement accentuée à la base du placentôme, au niveau de la mince assise de conjonctif lâche située entre le " berceau " et le muscle utérin. Certains des vaisseaux irrigant le " berceau " et les axes villositaires présentent une diminution de leur calibre au tiers de la hauteur des villosités tandis que d'autres sont obturés par l'accolement de leurs parois.

Du côté fotal, les cellules prismatiques assurant l'engrènement avec le syncytium maternel présentent un réseau ergastoplasmique plus développé et un ensemble de granulations semblables à des lysosomes dans la partie apicale du cytoplasme.

\section{III. - Après parturition, mais avant l'expulsion du placenta}

(animaux du Groupe 3 abattus $I \mathrm{~h}$ 30 après la mise bas)

Du côté maternel, l'apparition d'un léger œedème perturbe l'orientation des fibres de collagène et de réticuline; l'occlusion des vaisseaux sanguins villositaires se poursuit tandis que de nombreuses villosités sont déchirées au tiers de leur hauteur. 
De plus des polynucléaires apparaissent dans les vaisseaux sanguins qui irriguent le conjonctif lâche utérin.

Du côté fœetal, les capillaires irrigant les villosités sont engorgées sans doute du fait de la stase sanguine. L'aspect ultrastructural des cellules géantes n'est pas modifié ; les microvillosités des cellules de la zone d'engrènement se rétractent et se désengrènent du tissu maternel. $\mathrm{A}$ ce niveau s'accumulent de très nombreux corps denses aux électrons de type lysosomal. Les organites d'une cellule peuvent être tous rassemblés dans un grand autolysosome (fig. 2). L'expulsion du placenta se produit environ 2 heures après la mise bas et à ce moment la séparation des tissus maternels et fœtaux est imparfaite et laisse des bribes de villosités fœtales dans les touffes caronculaires maternelles.

\section{IV. - Après l'expulsion du placenta}

(animaux du groupe 3 abattus I jour au moins après la mise bas)

24 heures après le part, l'utérus a considérablement involué ; de très nombreux polynucléaires se sont infiltrés dans le tissu conjonctif lâche. Ce dernier est gonflé par un œdème qui fait s'éverser le " berceau " et les villosités maternelles restantes, facilitant ainsi l'élimination des bribes persistantes (fig. 3).

30 heures après la mise bas, l'œdème est à son maximum de développement puis il commence à régresser.

72 heures après la délivrance, le caroncule est réduit à un disque plat essentiellement composé de tissu conjonctif d'où les polynucléaires ont disparu. La lumière utérine contient les résidus des lyses cellulaires qui ont un aspect rougeâtre.

Les Brebis et les Chèvres traitées par la Dexaméthasone délivrent normalement ; aucune différence avec les animaux témoins n'a été relevée dans l'évolution des tissus maternels et fotaux après la mise bas.

\section{DISCUSSION}

Nos observations montrent que l'aspect cytologique des zones intercotylédonnaires et des placentômes chez la Brebis et la Chèvre, plus de 28 heures avant la mise bas, est identique à celui qui a été décrit à mi-gestation par WrMSATT (I950, I95I), Drieux et Thierry (I95 I b), Björkman (I962, I965), LAWN et al. (1963), VAN WyK et al. (1972) chez les ovidés.

Les glandes utérines sont actives au terme comme en début de gestation (HOYES, r972). En fin de gestation, le rôle trophique de ces glandes est discutable; il n'en reste pas moins que les cellules foetales de la zone intercotylédonnaire sont revếues de microvillosités apicales, ce qui témoigne en faveur d'une fonction d'absorption.

Jusqu'au terme on observe également les signes d'une intense phagocytose avec digestion des globules rouges maternels par les cellules foetales de la zone de résorption sanguine. Ceci peut être considéré comme un mode de transfert placentaire qui coexisterait pour le fer en particulier avec un passage direct sous forme 
conjuguée à une protéine mis en évidence sur la brebis (Hoskrns et HaNSARD, I964).

Ces structures placentaires apparaissent donc très stables tout au long de la gestation; les modifications ne se produisent qu'au cours des dernières heures et se précipitent dans les 2 heures post-partum, avant 1'expulsion du placenta.

Elles concernent tout d'abord le tissu conjonctif maternel et sa vascularisation ; puis elles se manifestent par la rétraction des microvillosités de la zone d'engrènement, la multiplication des corps " lysosomaux " maternels et fotaux sans doute induite par les œstrogènes (BRIGGS, I973; LIGGINS, I973), et la dégénérescence de nombreuses cellules fœtales. Toutes ces modifications font suite à l'élévation brutale des œstrogènes libres, de la prostaglandine $\mathrm{F}_{2}$ et de la chute de la progestérone (CuRRIE et al., I972; THORBURN et al., I972; LigGINs et al., I973 ; LIGGINS, I973) dont résultent les contractions du myomètre.

Ręu pour publication en octobre 1975.

\title{
SUMMARY
}

\author{
HISTOLOGICAL, STUDY OF THE PLACENTA IN EWE,
}

AND GOAT AT TIME OF BIRTH

Inducing birth in ewe and goat with dexamethasone does not seem to change fine structura development of the placenta and endometrium. This placental tissue evolution appears in the last day of pregnancy.

There is maternal dissociation of dense caruncular connective tissue and loose uterine connective tissue, accompanied by changes in villi vasculature. Simultaneously, there is fetal multiplication of lysosomal-type structures and degeneration of some cells. These changes become pronounced very soon after birth before the placenta is expulsed. Polynuclear cells are found in blood vessels of uterine connective tissue which becomes slightly oedematous. After delivery, fetal tissue retained in the caruncular tufts is completely eliminated as a consequence of the developing oedema which leads to caruncular eversion and reaches a maximum 30 hours after birth.

\section{RÉFÉRENCES BIBLIOGRAPHIQUES}

ASDELL S. A., 1964. Patterns of mammalian reproduction. (Second Edition), Comstock publishing associates. The Cornell University Press, Ithaca N. Y., 623-630.

BJörkman N., 1956. Morphological studies on the epithelia of the intercotyledonnary component of the bovine placenta. Acta Morphol. Neer. Scand., 1, I-10.

Björkman N., I962. Ultrastructural features of the ovine placentome. I'th. Cong. for Electron. Microsc., Academic Press.

BJörkman N., 1965. Fine structure of the ovine placentome. J. Anat., 92, 283-297.

Bosc M. J., I974. La parturition provoquée chez les mammifères domestiques. I-24, in Avortement et parturition provoqués. Masson éd., Paris.

Briggs M. 1973. Lysosomal enzyme activation by steroid hormones in vivo. J. Steroid. Bioch., 4, $34 \mathrm{I}-347$.

Currie W.B., Wong M.F., Cox R. I., Thorburn G. D., I972. Spontaneous or Dexamethasone induced parturition in the sheep and goat: changes in plasma concentrations of maternal Prostaglandin $F$ and fetal oestrogens sulfate. Mem. Soc. Endocr., 20, 95-II8.

Drieux H., ThIERRY C., I95I a. Placentation chez les mammifères domestiques : III. Placenta des bovidés. Rec. Med. Vet. Alfort, 127, 5-25. 
Drieux H., Thierry C., I95 $b$. Placentation chez les mammifères domestiques : IV. Placenta des ovidés. Rec. Med. Vet. Alfort, 128, 5-18.

EDWARDS V.D., SimoN G.T., I970. Ultrastructural aspects of red cells destruction in the normal rat spleen. J. Litr. Res., 33, I87-201.

Hoskins F. H., Hansard S. L., I964. Placental transfer and fetal tissue iron utilization in sheep. J. Nutr., 88, 10-14.

Hoyes A. D., 1972. Endometrial glands of the pregnant sheep : ultrastructural study. J. Anat., 111, $55-67$.

Lawn A. M., Chiguoine A. D., Amoroso E. C., I963. The ultrastructure of the placenta of the sheep and the goat. J. Anat. (London), 97, 306.

Liggins G. C., Fairclough R. J., Grieves S., Kendall J. Z., Knox B. S., I973. The mecanism of initiation of parturition in the ewe. Rec. Progr. Horm. Res., 29, III-I50.

Liggins G. C., 1973. Parturition in the sheep and the human. Internat. Symp. on Physiologic and Genetic aspects of Reproduction. Salvador, Bahia, Brasil, Dec. 5-7.

Pavsic F., Skusek F. R., I97r. Probleme des Lösungsmechanismus der Plazenta beim Rind. Dt. Tierärztl. IWsch., 78, 106.

Steven D. H., 1975. Separation of the placenta in the ewe : an ultrastructural study. Quaterly $J$. Exp. Physiol., 60, 37-44.

Van Wyk L. C., VAN Niekerk C. H., Belonje P. C., I972. The histology of the placentome of the ewe before and during parturition. J. Sth. Afr. Vet. Assoc., 43, I3-I7.

Wimsatt W. A., 1950. New histological observations on the placenta of the sheep. Am. J.Anat., 87, 39 I- 456 .

Wimsatt W.A., 1951. Observations on the morphogenesis, cytochemistry and signifiance of the binucleate giant cells of the placenta of ruminants. Am.J. Anat., 89, 233-281. 
PLANCHE I

\section{I. - Epithellum fotal}

de la zone de résorption sanguine

$(\times 1700)$

Brebis abattue plus de $28 \mathrm{~h}$ avant la parturition.

Etapes de la digestion des globules rouges maternels phagocytés, de la zone apicale à la partie infranueléaire.

A : Apex cellutaire

CG : Cellule géante

GR : Globule rotige

L. : Lysosome* 


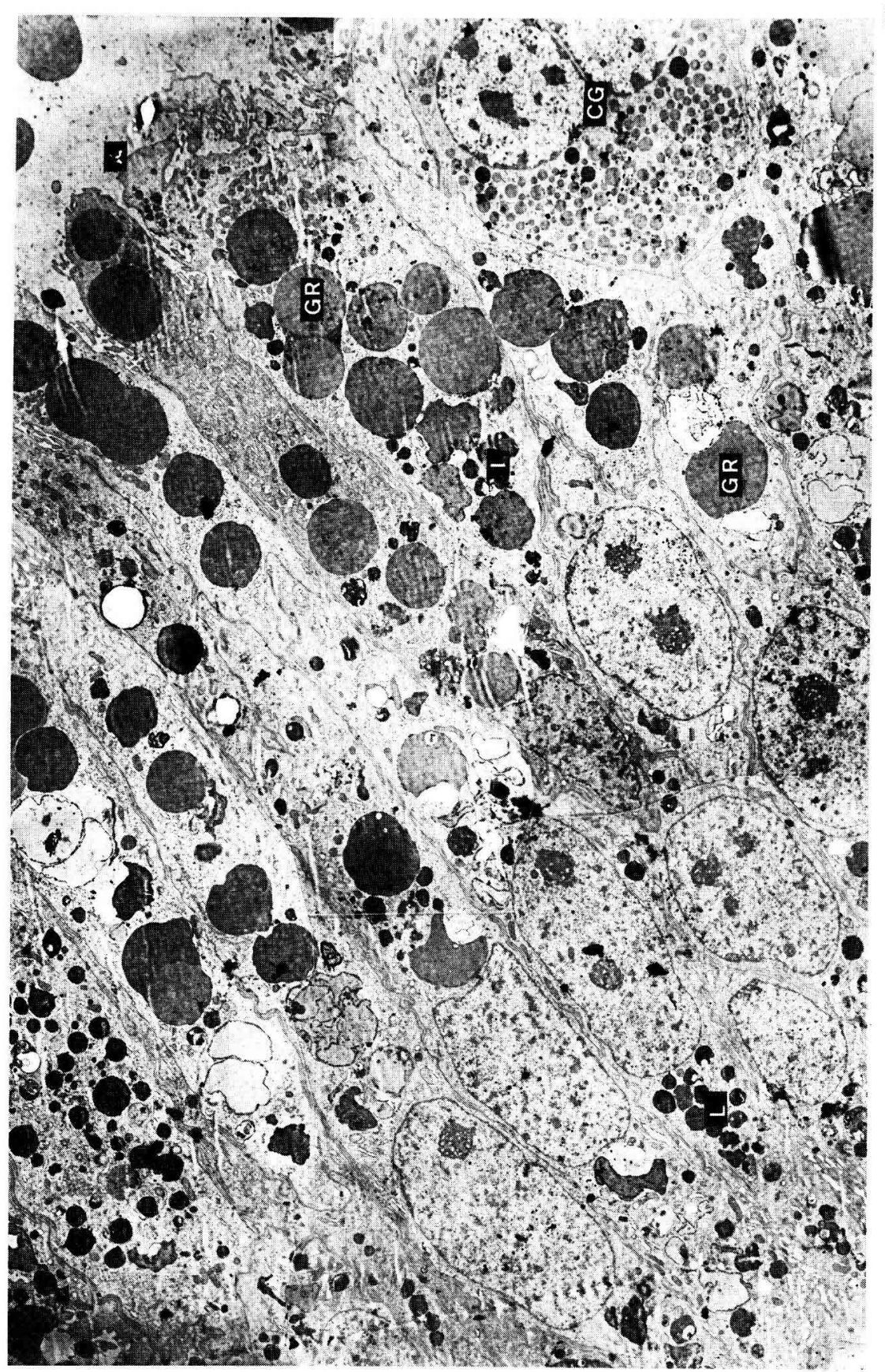


PLANCHE II

\section{2. - Cellule fotale de la zone d'engrènement.}

Brebis abattue 1 h 30 post-partum.

Noter la présence d'un autolysosome qui a phagocyté les organites cellulaires.

$(\times 5000)$.

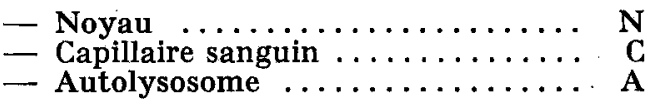

\section{3. - Caroncule.}

Chèvre abattue $24 \mathrm{~h}$ post-partum.

Noter la présence d'un œdème du tissu conjonctif qui fait s'éverser le caroncule.

- Caroncule ................... CAR

- Conjonctif dense du " berceau ......... CD

- Conjonctif lâche................ GL

- Glande utérine ................ GL UT

- Artériole utérine ................ A

— Epithélium utérin $\ldots \ldots \ldots \ldots \ldots \ldots \ldots \ldots \ldots$ EP UT 


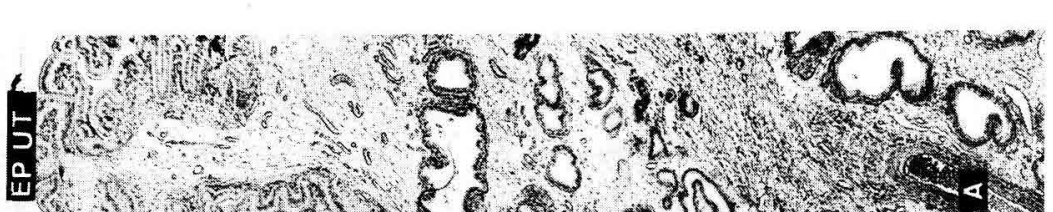

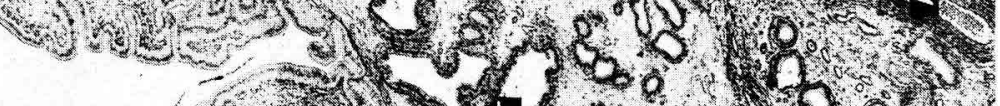
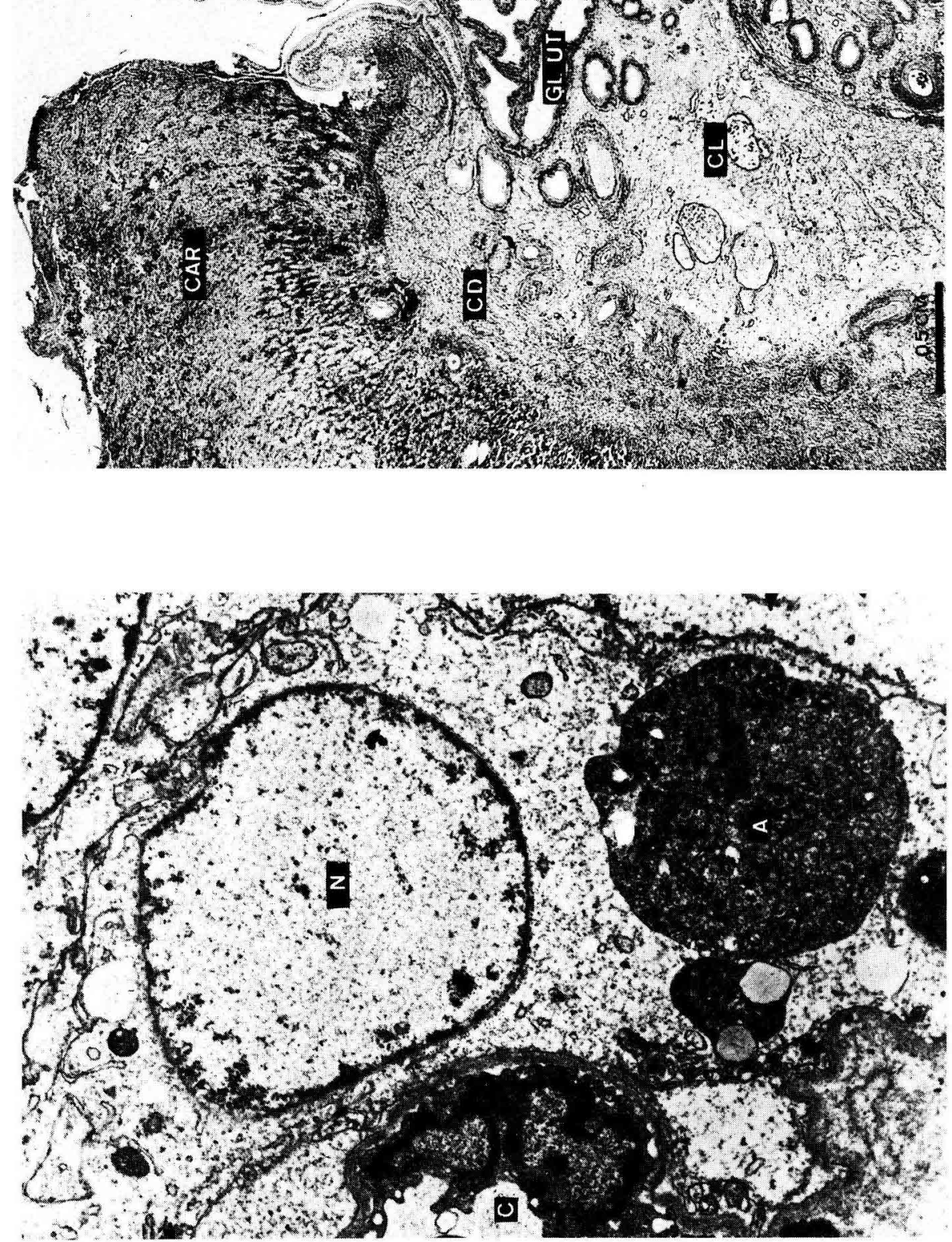